\title{
GNM ordered variational inequality system with ordered Lipschitz continuous mappings in an ordered Banach space
}

\author{
Hong-Gang Li1* Dong Qiu' and Maoming Jin²
}

"Correspondence: lihg12@126.com 'Applied Mathematics Institute, College of Mathematics and Physics, Chongqing University of Posts and Telecommunications, Chongqing, 400065, China Full list of author information is available at the end of the article

\begin{abstract}
The main purpose of this paper is to introduce and study a new class of generalized nonlinear mixed ordered variational inequalities systems with ordered Lipschitz continuous mappings in ordered Banach spaces. Then, applying the matrix analysis and the vector-valued mapping fixed point analysis method, an existence theorem of solutions for this kind of the system is established. Furthermore, based on the existence theorem and the new ordered $B$-restricted-accretive mappings, a general algorithm for solving the systems is introduced and applied to the approximation solvability of the systems on hand. The obtained results seem to be general in nature. MSC: 49J40;47H06

Keywords: general nonlinear mixed ordered variational inequality system; ordered Lipschitz continuous mappings; $B$-restricted-accretive mappings; iterative algorithm; convergence; ordered Banach space
\end{abstract}

\section{Introduction}

Let $X$ be a real ordered Banach space with a norm $\|\cdot\|$, a zero $\theta$, a normal cone $\mathbf{P}$, a normal constance $N$ of $\mathbf{P}$ and a partial ordered relation $\leq$ defined by the cone $\mathbf{P}$. Let $F_{i j}, g, f$ : $X \times X \rightarrow X$ be single-valued nonlinear ordered compression mappings, and let $F_{i j}$ be a Lipschitz continuous mapping $(i, j=1,2)$, and for any $x, y \in X$,

$$
\operatorname{range}(f) \cap \operatorname{dom}\left(F_{11}(\cdot, y)\right) \cap \operatorname{range}(g) \cap \operatorname{dom}\left(F_{22}(x, \cdot)\right) \neq \emptyset,
$$

we consider the following problem:

For $u, v \in X$, find $x, y \in X$ such that

$$
\left\{\begin{array}{l}
u \leq F_{11}(f(x), y)+F_{12}(y, x), \\
v \leq F_{21}(x, y) \oplus F_{22}(x, g(y)),
\end{array}\right.
$$

which is called a generalized nonlinear mixed ordered variational inequality system (GNM ordered variational inequality system) with ordered Lipschitz continuous mappings in an ordered Banach space. Obviously, system (1.1) belongs to a new class of generalized nonlinear mixed ordered variational inequality systems with the $\oplus$ calculation.

\section{Springer}

๑2013 Li et al.; licensee Springer. This is an Open Access article distributed under the terms of the Creative Commons Attribution License (http://creativecommons.org/licenses/by/2.0), which permits unrestricted use, distribution, and reproduction in any medium, provided the original work is properly cited. 
For a suitable choice of the mappings $u, v, f, g, F_{i j}(i, j=1,2)$ and the space $X$, a number of known classes of ordered variational inequalities, which have been studied by the authors as special cases of system (1.1) in the Banach space (see [1, 2]).

Remark 1.1 Some special cases of system (1.1):

(1) Let $F_{12}(\cdot, \cdot)=F_{21}(\cdot, \cdot)=F_{22}(\cdot, \cdot)=0$ be zero operators, $u=v=\theta$ and $F_{11}(f(x), y)=A(x)$ for any $y \in X$, then system (1.1) becomes the following problem: Find $x \in X$ such that

$$
\theta \leq A(f(x))
$$

which is called a generalized nonlinear ordered variational inequality (a generalized nonlinear ordered equation, as changed $\geq$ to $=$ ) in an ordered Banach space (see [1]).

(2) Let $F_{11}(\cdot, \cdot)=F_{12}(\cdot, \cdot)=0$ be zero operators, $F_{21}(f(x), y)=A(x), u=v=\theta$ and $F_{22}(x, g(y))=F(x, g(x))$ for any $y=x$, then system (1.1) becomes the following problem: Find $x \in X$ such that

$$
\theta \leq A(x) \oplus F(x ; g(x))
$$

which is called a new class of general nonlinear ordered variational inequality (a general nonlinear ordered equation, as changed $\geq$ to $=$ ) in an ordered Banach space (see [2]).

(3) Let $F_{21}(\cdot, \cdot)=F_{22}(\cdot, \cdot)=0$ be zero operators and $u=v=\theta$, then system (1.1) becomes the following problem: Find $x, y \in X$ such that

$$
\theta \leq F_{11}(f(x), y)+F_{12}(y, x)
$$

which is studied by many authors in a Banach space (see [3] et al.).

In recent years, though we have succeeded in the area of studies of variational inequality (inclusion) systems, yet, the studies of ordered variational inequality (inclusion) systems are beginning in very recent research works on an ordered Banach space (see $[1,2,4-$ 9]). From 1999 till present, some new and interesting problems for systems of variational inequalities (inclusions) have been introduced and studied in this field (see [1-32]).

Very recently, the approximation solution for general nonlinear ordered variational inequalities and ordered equations $[1,2]$ and a nonlinear ordered inclusion problem $[8,9]$ have been studied by Li in an ordered Banach space. For details, we refer the reader to [1-32] and the references therein.

\section{Preliminaries}

We need to recall the following concepts and results for solving system (1.1).

Definition 2.1 [21] Let $X$ be a real ordered Banach space with a norm $\|\cdot\|$, a normal cone $\mathbf{P}$ and a partial ordered relation $\leq$ defined by the cone $\mathbf{P}$, for $x, y \in X$, if $x \leq y$ (or $y \leq x)$ holds, then $x$ and $y$ are said to be a comparison between each other (denoted by $x \propto y$ for $x \leq y$ and $y \leq x$ ). 
Lemma 2.2 [1] Let $X$ be a real ordered Banach space with a norm $\|\cdot\|$, a normal cone $\mathbf{P}$ and a partial ordered relation $\leq$ defined by the cone $\mathbf{P}$, for arbitrary $x, y \in X, \operatorname{lub}\{x, y\}$ and $\operatorname{glb}\{x, y\}$ express the least upper bound of the set $\{x, y\}$ and the greatest lower bound of the set $\{x, y\}$ on the partial ordered relation $\leq$, respectively. Suppose that $\operatorname{lub}\{x, y\}$ and $\operatorname{glb}\{x, y\}$ exist, some binary operators can be defined as follows:

(1) $x \vee y=\operatorname{lub}\{x, y\}$

(2) $x \wedge y=\operatorname{glb}\{x, y\}$

(3) $x \oplus y=(x-y) \vee(y-x)$.

$\vee, \wedge$, and $\oplus$ are called $O R, A N D$, and XOR operations, respectively. For arbitrary $x, y, w \in$ $X$, the following relations hold:

(1) $x \oplus y=y \oplus x$

(2) $x \oplus x=\theta$;

(3) $\theta \leq x \oplus \theta$;

(4) let $\lambda$ be real, then $(\lambda x) \oplus(\lambda y)=|\lambda|(x \oplus y)$;

(5) if $x, y$, and $w$ can be compared with each other, then

$$
(x \oplus y) \leq x \oplus w+w \oplus y
$$

(6) let $(x+y) \vee(u+v)$ exist, and if $x \propto u$,v and $y \propto u, v$, then

$$
(x+y) \oplus(u+v) \leq(x \oplus u+y \oplus v) \wedge(x \oplus v+y \oplus u) ;
$$

(7) if $x, y, z, w$ can be compared with each other, then

$$
(x \wedge y) \oplus(z \wedge w) \leq((x \oplus z) \vee(y \oplus w)) \wedge((x \oplus w) \vee(y \oplus z))
$$

(8) $\alpha x \oplus \beta x=|\alpha-\beta| x=(\alpha \oplus \beta) x$, if $x \propto \theta$.

Lemma 2.3 [5] If $x \propto y$, then $\operatorname{lub}\{x, y\}$, and $\operatorname{glb}\{x, y\}$ exist, $x-y \propto y-x$, and $\theta \leq(x-y) \vee$ $(y-x)$

Lemma 2.4 [5] If for any natural number $n, x \propto y_{n}$, and $y_{n} \rightarrow y^{*}(n \rightarrow \infty)$, then $x \propto y^{*}$.

Lemma 2.5 Let $X$ be a real ordered Banach space with a norm $\|\cdot\|$, a zero $\theta$, a normal cone $\mathbf{P}$, a normal constance $N$ of $\mathbf{P}$ and a partial ordered relation $\leq$ defined by the cone $\mathbf{P}$. $A: X \rightarrow X$ is comparative, then for $x, y \in X$, if $x \propto y$ [1], then

(1) $\|x \vee y\| \leq\|x\| \vee\|y\| \leq\|x\|+\|y\|$,

(2) $\|x \oplus y\|=\|x-y\| \leq N\|x \oplus y\|$,

(3) $\lim _{x \rightarrow x_{0}}\left\|A(x)-A\left(x_{0}\right)\right\|=0$ if and only if $\lim _{x \rightarrow x_{0}} A(x) \oplus A\left(x_{0}\right)=\theta$.

Proof Result (1) is obvious; (2) follows from (1), Definition 2.2 in [1], Lemma 2.7 in [2]; (3) follows from (1) and (2). This completes the proof.

Definition 2.6 Let $X$ be a real ordered Banach space, and let $F: X \times X \rightarrow X$ be a mapping. The operator $F: X \times X \rightarrow X$ is said to be an ordered Lipschitz continuous with constants $(\mu, v)$ if $x \propto y, u \propto v$, then $F(u, x) \propto F(v, y)$, and there exist constants $\mu, v>0$ such that

$$
F(u, x) \oplus F(v, y) \leq \mu(u \oplus v)+v(x \oplus y) .
$$


Definition 2.7 [2] Let $X$ be a real ordered Banach space, let $B: X \rightarrow X$ be a mapping, and let $I$ be an identity mapping on $X$. A mapping $A: X \rightarrow X$ is said to be a $B$-restrictedaccretive mapping if $A, B$ and $A \wedge B: x \in X \rightarrow A(x) \wedge B(x) \in X$ all are comparisons, and they are comparisons with each other, and there exist two constants $0<\alpha_{1}, \alpha_{2} \leq 1$ such that for arbitrary $x, y \in X$,

$$
\begin{aligned}
& (A(x) \wedge B(x)+I(x)) \oplus(A(y) \wedge B(y)+I(y)) \\
& \quad \leq \alpha_{1}((A(x) \wedge B(x)) \oplus(A(y) \wedge B(y)))+\alpha_{2}(x \oplus y)
\end{aligned}
$$

holds, where $I$ is an identity mapping on $X$.

Definition 2.8 Let $X$ be a real ordered Banach space with a norm $\|\cdot\|$, a zero $\theta$, a normal cone $\mathbf{P}$, a normal constance $N$ of $\mathbf{P}$ and a partial ordered relation $\leq$ defined by the cone $\mathbf{P}$. If $X \times X$ is a product Banach space with the normal $\|\cdot\|$ and an ordered relation $\leq$, and the following conditions are satisfied:

(1) $\|(x, y)\|=\max \{\|x\|,\|y\|\}$ for any $(x, y) \in X \times X$;

(2) $\left(x_{1}, y_{1}\right) \propto\left(x_{2}, y_{2}\right)$ if and only if $x_{1} \propto x_{2}, y_{1} \propto y_{2}$, and $\left(x_{1}, y_{1}\right) \leq\left(x_{2}, y_{2}\right)$ if and only if $x_{1} \leq x_{2}, y_{1} \leq y_{2}$ in $X$

(3)

$$
\begin{aligned}
& \left(x_{1}, y_{1}\right) \vee\left(x_{2}, y_{2}\right)=\left(x_{1} \vee x_{2}, y_{1} \vee y_{2}\right), \\
& \left(x_{1}, y_{1}\right) \wedge\left(x_{2}, y_{2}\right)=\left(x_{1} \wedge x_{2}, y_{1} \wedge y_{2}\right), \\
& \left(x_{1}, y_{1}\right) \oplus\left(x_{2}, y_{2}\right)=\left(x_{1} \oplus x_{2}, y_{1} \oplus y_{2}\right) .
\end{aligned}
$$

Then $X \times X$ is called an ordered product Banach space.

Definition 2.9 Let $X$ be a real ordered Banach space with a norm $\|\cdot\|$, a zero $\theta$, a normal cone $\mathbf{P}$, a normal constance $N$ of $\mathbf{P}$ and a partial ordered relation $\leq$ defined by the cone $\mathbf{P}$. Let $X \times X$ be an ordered product Banach space. For a vector-valued mapping $\vec{G}=\left(G_{1}, G_{2}\right)\left(\right.$ or $\left.\left(G_{1}, G_{2}\right)^{T}\right): X \times X \rightarrow X \times X$ in $X \times X$, if there exists a point $\left(x^{*}, y^{*}\right) \in X \times X$ such that

$$
\vec{G}\left(x^{*}, y^{*}\right)=\left(G_{1}, G_{2}\right)\left(x^{*}, y^{\prime \prime}\right)=\left(x^{*}, y^{*}\right),
$$

then $\left(x^{*}, y^{*}\right)$ is called a fixed point of vector-valued mapping $\vec{G}$ in ordered product Banach space.

The following results are obvious.

Lemma 2.10 Let $X$ be a real ordered Banach space with a norm $\|\cdot\|$, a zero $\theta$, a normal cone $\mathbf{P}$, a normal constance $N$ of $\mathbf{P}$ and a partial ordered relation $\leq$ defined by the cone $\mathbf{P}$. Let $X \times X$ be an ordered product Banach space. For sequences $\left\{x_{n}\right\}$ and $\left\{y_{n}\right\}$ in $X$, in $X \times X$,

$$
\left(x_{n}, y_{n}\right) \rightarrow\left(x^{*}, y^{*}\right) \quad \text { if and only if } x_{n} \rightarrow x^{*} \quad \text { and } \quad y_{n} \rightarrow y^{*} \quad \text { as } n \rightarrow \infty \text {. }
$$

Lemma 2.11 Let $X$ be a real ordered Banach space with a norm $\|\cdot\|$, a zero $\theta$, a normal cone $\mathbf{P}$, a normal constance $N$ of $\mathbf{P}$ and a partial ordered relation $\leq$ defined by the cone $\mathbf{P}$. Let 
$X \times X$ bean ordered product Banach space. Let $\vec{G}=\left(G_{1}, G_{2}\right)\left(\right.$ or $\left.\left(G_{1}, G_{2}\right)^{T}\right): X \times X \rightarrow X \times X$ be a vector-valued mapping in $X \times X$ if for any $\left(x_{i}, y_{i}\right) \in X \times X(i=1,2),\left(x_{1}, y_{1}\right) \propto\left(x_{2}, y_{2}\right)$, and there exist a constance $1>\delta>0$ such that

$$
\left\|\left(G_{1}, G_{2}\right)\left(x_{1}, y_{1}\right) \oplus\left(G_{1}, G_{2}\right)\left(x_{2}, y_{2}\right)\right\| \leq \delta\left\|\left(x_{1}, y_{1}\right) \oplus\left(x_{2}, y_{2}\right)\right\|,
$$

then $\left(G_{1}, G_{2}\right)$ has a fixed point in $X \times X$.

Proof This directly follows from Lemma 2.2, Lemma 2.5(2) and the contraction mapping principle.

\section{Approximation solution for GNM system (1.1)}

In this section, we will change from the solution of system (1.1) to finding a fixed point for a vector-valued mapping, and by using the vector-valued mapping fixed point analysis method, show the convergence of the approximation sequences of the solution for system (1.1) in an ordered product Banach space.

Lemma 3.1 Let $X$ be a real ordered Banach space with a norm $\|\cdot\|$, a zero $\theta$, a normal cone $\mathbf{P}$, a normal constance $N$ of $\mathbf{P}$ and a partial ordered relation $\leq$ defined by the cone $\mathbf{P}$, and let $X \times X$ be an ordered product Banach space. If $g, f, B_{i}: X \rightarrow X$ are ordered compressions, $F_{i j}$ : $X \times X \rightarrow X$ is order $\left(\mu_{i j}, v_{i j}\right)$-Lipschitz continuous, and let $g, f, B_{1}, B_{2}$ and $F_{i j}$ be comparison mappings with each other (where $i, j=1,2)$. Then system (1.1) has a solution $\left(x^{*}, y^{*}\right)$ if and only if there exist two ordered compressions $B_{1}$ and $B_{2}$ such that the vector-valued mapping $\vec{G}=\left(G_{1}(x, y), G_{2}(x, y)\right): X \times X \rightarrow X \times X$,

$$
\begin{aligned}
& G_{1}(x, y)=\left(F_{11}(f(x), y)+F_{12}(y, x)-u\right) \wedge B_{1}(x)+I(x) \text { and } \\
& G_{2}(x, y)=\left(F_{21}(x, y) \oplus F_{22}(x, g(y))-v\right) \wedge B_{2}(y)+I(y),
\end{aligned}
$$

has the fixed point $\left(x^{*}, y^{*}\right)$ in an ordered Banach space $X \times X$.

Proof Let $\left(x^{*}, y^{*}\right)$ be a fixed point of the vector-valued mapping (3.1), then, obviously, $\left(x^{*}, y^{*}\right)$ is a solution of system (1.1).

On the other hand, choosing

$$
B_{1}(x)= \begin{cases}\theta, & \text { if } \theta \leq F_{11}(f(x), y)+F_{12}(y, x)-u, \\ \zeta_{1} x, & \text { otherwise }\end{cases}
$$

and

$$
B_{2}(y)= \begin{cases}\theta, & \text { if } \theta \leq F_{21}(x, y) \oplus F_{22}(x, g(y))-v, \\ \zeta_{2} y, & \text { otherwise }\end{cases}
$$

where $1>\zeta_{1}, \zeta_{2}>0$, if $\left(x^{*}, y^{*}\right)$ is a solution of system (1.1), then by using [1, 2],

$$
\begin{aligned}
& \left(F_{11}\left(f\left(x^{*}\right), y^{*}\right)+F_{12}\left(y^{*}, x^{*}\right)-u\right) \wedge B_{1}\left(x^{*}\right)+I\left(x^{*}\right)=x^{*}, \\
& \left(F_{21}\left(x^{*}, y^{*}\right) \oplus F_{22}\left(x^{*}, g\left(y^{*}\right)\right)-v\right) \wedge B_{2}\left(y^{*}\right)+I\left(y^{*}\right)=y^{*}
\end{aligned}
$$


hold. Therefore, $\left(x^{*}, y^{*}\right)$ is a fixed point of the vector-valued mapping (3.1), where the mappings $B_{1}$ and $B_{2}$ are ordered compressions [2]. This completes the proof.

Theorem 3.2 Let $X$ be a real ordered Banach space with a norm $\|\cdot\|$, a zero $\theta$, a normal cone $\mathbf{P}$, a normal constance $N$ of $\mathbf{P}$ and a partial ordered relation $\leq$ defined by the cone $\mathbf{P}$, and let $X \times X$ be an ordered product Banach space. Let $g$ and $f$ be ordered compressions with respect to $\gamma_{g}$ and $\gamma_{f}$, respectively; let $B_{i}: X \rightarrow X$ be an ordered compression mapping with $\zeta_{i}$, let $F_{i j}: X \times X \rightarrow X$ be an ordered $\left(\mu_{i j}, v_{i j}\right)$-Lipschitz continuous $(i, j=1,2)$, and let $g, f, B_{1}, B_{2}$ and $F_{i j}(i, j=1,2)$ be comparison mappings with each other. If $F_{11}+F_{12}-u$ is a $B_{1}$-restricted-accretive mapping with $\left(\alpha_{1}, \alpha_{2}\right)$, and $F_{21} \oplus F_{22}-v$ is a $B_{2}$-restricted-accretive mapping with $\left(\beta_{1}, \beta_{2}\right)$, and

$$
\begin{array}{r}
N \max \left\{\alpha_{1}\left(\mu_{11} \gamma_{f}+v_{12}+\zeta_{1}\right)+\alpha_{2}, \alpha_{1}\left(v_{11}+\mu_{12}\right),\right. \\
\left.\beta_{1}\left(\mu_{21} \oplus \mu_{22}\right),\left(\beta_{1} \nu_{21} \oplus v_{22} \gamma_{g}\right)+\zeta_{2}+\beta_{2}\right\}<1
\end{array}
$$

holds, then for the general nonlinear mixed ordered variational inequality system (1.1), there exists a solution $\left(x^{\prime \prime}, y^{\prime \prime}\right)$.

Proof Let $X$ be a real ordered Banach space, and let $X \times X$ be an ordered product Banach space. Setting

$$
\begin{aligned}
& G_{1}(x, y)=\left(F_{11}(f(x), y)+F_{12}(y, x)-u\right) \wedge B_{1}(x)+I(x), \\
& G_{2}(x, y)=\left(F_{21}(x, y) \oplus F_{22}(x, g(y))-v\right) \wedge B_{2}(y)+I(y) .
\end{aligned}
$$

Since $F_{11}+F_{12}-u$ is a $B_{1}$-restricted-accretive mapping with $\left(\alpha_{11}, \alpha_{12}\right), F_{21} \oplus F_{22}-v$ is a $B_{2}$ restricted-accretive mapping with $\left(\beta_{11}, \beta_{12}\right)$, and $F_{11}$ and $F_{12}$ are ordered $\left(\mu_{11}, v_{11}\right)$-Lipschitz continuous and $\left(\mu_{12}, v_{12}\right)$-Lipschitz continuous, respectively, then for any given $x_{i}, y_{j} \in X$ and $x_{i} \propto y_{j}(i, j=1,2)$, by Lemma 2.2(6), (7), Definition 2.7 and [1,2], we can obtain the following inequalities:

$$
\begin{aligned}
\theta \leq & G_{1}\left(x_{1}, y_{1}\right) \oplus G_{1}\left(x_{2}, y_{2}\right) \\
= & \left(\left(F_{11}\left(f\left(x_{1}\right), y_{1}\right)+F_{12}\left(y_{1}, x_{1}\right)-u\right) \wedge B_{1}\left(x_{1}\right)+I\left(x_{1}\right)\right) \\
& \oplus\left(\left(F_{11}\left(f\left(x_{2}\right), y_{2}\right)+F_{12}\left(y_{2}, x_{2}\right)-u\right) \wedge B_{1}\left(x_{2}\right)+I\left(x_{2}\right)\right) \\
\leq & \alpha_{1}\left(\left(\left(F_{11}\left(f\left(x_{1}\right), y_{1}\right)+F_{12}\left(y_{1}, x_{1}\right)-u\right) \wedge B_{1}\left(x_{1}\right)\right)\right. \\
& \left.\oplus\left(\left(F_{11}\left(f\left(x_{2}\right), y_{2}\right)+F_{12}\left(y_{2}, x_{2}\right)\right)-u\right) \wedge B_{1}\left(x_{2}\right)\right)+\alpha_{2}\left(x_{1} \oplus x_{2}\right) \\
\leq & \alpha_{11}\left(\left(\left(F_{11}\left(f\left(x_{1}\right), y_{1}\right)+F_{12}\left(y_{1}, x_{1}\right)-u\right)\right.\right. \\
& \left.\left.\oplus\left(F_{11}\left(f\left(x_{2}\right), y_{2}\right)+F_{12}\left(y_{2}, x_{2}\right)\right)-u\right) \vee\left(B_{1}\left(x_{1}\right) \oplus B_{1}\left(x_{2}\right)\right)\right)+\alpha_{2}\left(x_{1} \oplus x_{2}\right) \\
\leq & \alpha_{1}\left(\left(\left(F_{11}\left(f\left(x_{1}\right), y_{1}\right) \oplus F_{11}\left(f\left(x_{2}\right), y_{2}\right)\right)\right.\right. \\
& \left.\left.+\left(\left(F_{12}\left(y_{1}, x_{1}\right)-u\right) \oplus\left(F_{12}\left(y_{2}, x_{2}\right)-u\right)\right) \vee \zeta_{1}\left(x_{1} \oplus x_{2}\right)\right)\right)+\alpha_{2}\left(x_{1} \oplus x_{2}\right) \\
\leq & \alpha_{1}\left(\left(\left(F_{11}\left(f\left(x_{1}\right), y_{1}\right) \oplus F_{11}\left(f\left(x_{2}\right), y_{2}\right)\right)\right.\right. \\
& \left.\left.+\left(F_{12}\left(y_{1}, x_{1}\right) \oplus F_{12}\left(y_{2}, x_{2}\right)\right)\right) \vee \zeta_{1}\left(x_{1} \oplus x_{2}\right)\right)+\alpha_{2}\left(x_{1} \oplus x_{2}\right)
\end{aligned}
$$




$$
\begin{aligned}
\leq & \left(\alpha_{1}\left(\mu_{11} \gamma_{f}\left(x_{1} \oplus x_{2}\right)+v_{11}\left(y_{1} \oplus y_{2}\right)\right)\right. \\
& \left.+\alpha_{1}\left(\mu_{12}\left(y_{1} \oplus y_{2}\right)+v_{12}\left(x_{1} \oplus x_{2}\right)\right)\right) \vee \zeta_{1}\left(x_{1} \oplus x_{2}\right)+\alpha_{2}\left(x_{1} \oplus x_{2}\right) \\
\leq & \left(\alpha_{1}\left(\left(\mu_{11} \gamma_{f}+v_{12}\right)+\zeta_{1}\right)+\alpha_{2}\right)\left(x_{1} \oplus x_{2}\right)+\alpha_{1}\left(v_{11}+\mu_{12}\right)\left(y_{1} \oplus y_{2}\right)
\end{aligned}
$$

and

$$
\begin{aligned}
\theta \leq & G_{2}\left(x_{1}, y_{1}\right) \oplus G_{2}\left(x_{2}, y_{2}\right) \\
= & \left(\left(F_{21}\left(x_{1}, y_{1}\right) \oplus F_{22}\left(x_{1}, g\left(y_{1}\right)\right)-v\right) \wedge B_{2}\left(y_{1}\right)+I\left(y_{1}\right)\right) \\
& \oplus\left(\left(F_{21}\left(x_{2}, y_{2}\right) \oplus F_{22}\left(x_{2}, g\left(y_{2}\right)\right)-v\right) \wedge B_{2}\left(y_{2}\right)+I\left(y_{2}\right)\right) \\
\leq & \beta_{1}\left(\left(\left(\left(F_{21}\left(x_{1}, y_{1}\right) \oplus F_{21}\left(x_{2}, y_{2}\right)\right)-v\right) \wedge B_{2}\left(y_{1}\right)\right)\right. \\
& \left.\oplus\left(\left(F_{22}\left(x_{1}, g\left(y_{1}\right)\right) \oplus F_{22}\left(x_{2}, g\left(y_{2}\right)\right)\right)-v\right) \wedge B_{2}\left(y_{2}\right)\right)+\beta_{2}\left(y_{1} \oplus y_{2}\right) \\
\leq & \beta_{1}\left(\left(\left(\left(F_{21}\left(x_{1}, y_{1}\right) \oplus F_{21}\left(x_{2}, y_{2}\right)\right)\right)\right.\right. \\
& \left.\left.\oplus\left(F_{22}\left(x_{1}, g\left(y_{1}\right)\right) \oplus F_{22}\left(x_{2}, g\left(y_{2}\right)\right)\right)\right) \vee\left(B_{2}\left(y_{1}\right) \oplus B_{2}\left(y_{2}\right)\right)\right)+\beta_{2}\left(y_{1} \oplus y_{2}\right) \\
\leq & \beta_{1}\left(\left(\left(\left(F_{21}\left(x_{1}, y_{1}\right) \oplus F_{21}\left(x_{2}, y_{2}\right)\right)\right)\right.\right. \\
& \left.\left.\oplus\left(F_{22}\left(x_{1}, g\left(y_{1}\right)\right) \oplus F_{22}\left(x_{2}, g\left(y_{2}\right)\right)\right)\right) \vee \zeta_{2}\left(y_{1} \oplus y_{2}\right)\right)+\beta_{2}\left(y_{1} \oplus y_{2}\right) \\
\leq & \beta_{1}\left(\left(\left(\mu_{21}\left(x_{1} \oplus x_{2}\right)+v_{21}\left(y_{1} \oplus y_{2}\right)\right)\right.\right. \\
& \left.\left.\oplus\left(\mu_{22}\left(x_{1} \oplus x_{2}\right)+v_{22} \gamma_{g}\left(y_{1} \oplus y_{2}\right)\right)\right) \vee \zeta_{2}\left(y_{1} \oplus y_{2}\right)\right)+\beta_{2}\left(y_{1} \oplus y_{2}\right) \\
\leq & \beta_{1}\left(\left(\left(\mu_{21}\left(x_{1} \oplus x_{2}\right) \oplus \mu_{22}\left(x_{1} \oplus x_{2}\right)\right)\right.\right. \\
& \left.\left.+\left(v_{21}\left(y_{1} \oplus y_{2}\right) \oplus v_{22} \gamma_{g}\left(y_{1} \oplus y_{2}\right)\right)\right) \vee \zeta_{2}\left(y_{1} \oplus y_{2}\right)\right)+\beta_{2}\left(y_{1} \oplus y_{2}\right) \\
\leq & \beta_{1}\left(\mu_{21} \oplus \mu_{22}\right)\left(x_{1} \oplus x_{2}\right)+\left(\left(\beta_{1} v_{21} \oplus v_{22} \gamma_{g}\right)+\zeta_{2}+\beta_{2}\right)\left(y_{1} \oplus y_{2}\right) ;
\end{aligned}
$$

and

$$
\left(G_{1}, G_{2}\right)\left(x_{1}, y_{1}\right) \oplus\left(G_{1}, G_{2}\right)\left(x_{2}, y_{2}\right) \leq \boldsymbol{\Psi}\left(x_{1}, y_{1}\right) \oplus\left(x_{2}, y_{2}\right),
$$

where

$$
\boldsymbol{\Psi}=\left(\begin{array}{cc}
\alpha_{1}\left(\mu_{11} \gamma_{f}+v_{12}+\zeta_{1}\right)+\alpha_{2} & \alpha_{1}\left(\nu_{11}+\mu_{12}\right) \\
\beta_{1}\left(\mu_{21} \oplus \mu_{22}\right) & \left(\beta_{1} v_{21} \oplus v_{22} \gamma_{g}\right)+\zeta_{2}+\beta_{2}
\end{array}\right)
$$

Further, by [27] and Definition 2.2 in [1], we have

$$
\left\|\left(G_{1}, G_{2}\right)\left(x_{1}, y_{1}\right) \oplus\left(G_{1}, G_{2}\right)\left(x_{2}, y_{2}\right)\right\| \leq N\|\boldsymbol{\Psi}\|\left\|\left(x_{1}, y_{1}\right) \oplus\left(x_{2}, y_{2}\right)\right\|,
$$

where

$$
\begin{aligned}
\|\boldsymbol{\Psi}\|= & \max \left\{\alpha_{1}\left(\mu_{11} \gamma_{f}+v_{12}+\zeta_{1}\right)+\alpha_{2}, \alpha_{1}\left(\nu_{11}+\mu_{12}\right),\right. \\
& \left.\beta_{1}\left(\mu_{21} \oplus \mu_{22}\right),\left(\beta_{1} v_{21} \oplus v_{22} \gamma_{g}\right)+\zeta_{2}+\beta_{2}\right\},
\end{aligned}
$$

and $N$ is a normal constant of $\mathbf{P}$. 
It follows from (3.8) and the assumption condition (3.2) that $0<N\|\Psi\|<1$, and hence the vector-valued mapping

$$
\left(G_{1}, G_{2}\right)^{T}=\left(\left(\left(F_{11}(f, \cdot)+F_{12}(\cdot, \cdot)-u\right) \wedge B_{1}+I(\cdot),\left(F_{21}(\cdot, \cdot) \oplus F_{22}(\cdot, g)\right)-v\right) \vee B_{2}+I(\cdot)\right)^{T}
$$

has a fixed point $\left(x^{*}, y^{*}\right)$ for Lemma 2.11 , in an ordered Banach space $X \times X$, which is a solution for system (1.1) by Lemma 3.1. This completes the proof.

Theorem 3.3 Let the assumption conditions in Theorem 3.2 and (3.2) hold, that is,

$$
\begin{array}{r}
N \max \left\{\alpha_{1}\left(\mu_{11} \gamma_{f}+v_{12}\right)+\zeta_{1}+\alpha_{2}, \alpha_{1}\left(v_{11}+\mu_{12}\right),\right. \\
\left.\beta_{1}\left(\mu_{21} \oplus \mu_{22}\right),\left(\beta_{1} v_{21} \oplus v_{22} \gamma_{g}\right)+\zeta_{2}+\beta_{2}\right\}<1 .
\end{array}
$$

Then the iterative sequence $\left\{\left(x_{n}, y_{n}\right)\right\}$ generated by the following algorithm:

$$
\begin{aligned}
& x_{n+1}=(1-\rho) x_{n}+\rho\left(\left(F_{11}\left(f\left(x_{n}\right), y_{n}\right)+F_{12}\left(y_{n}, x_{n}\right)\right) \wedge B_{1}\left(x_{n}\right)+I\left(x_{n}\right)\right), \\
& y_{n+1}=(1-\sigma) y_{n}+\sigma\left(\left(\left(F_{21}\left(x_{n}, y_{n}\right) \oplus F_{22}\left(x_{n}, g\left(y_{n}\right)\right)\right) \wedge B_{2}\left(y_{n}\right)+I\left(y_{n}\right)\right)\right)
\end{aligned}
$$

for any $x_{0}, y_{0} \in X, x_{0} \propto y_{0},\left(x_{0}, y_{0}\right) \propto\left(x_{1}, y_{1}\right)$ and $1>\rho, \varrho>0$, converges strongly to $\left(x^{*}, y^{*}\right)$, which is a solution of system (1.1).

Proof Let the assumption conditions in Theorem 3.2 hold. For any given $x_{0}, y_{0} \in X$ and $x_{0} \propto y_{0},\left(x_{0}, y_{0}\right) \propto\left(x_{1}, y_{1}\right)$, setting

$$
\begin{aligned}
& G_{1}(x, y)=\left(F_{11}(f(x), y)+F_{12}(y, x)-u\right) \wedge B_{1}(x)+I(x), \\
& G_{2}(x, y)=\left(F_{21}(x, y) \oplus F_{22}(x, g(y))-v\right) \wedge B_{2}(y)+I(y),
\end{aligned}
$$

then for any $1>\rho, \sigma>0$, by algorithm (3.10), (3.4) and (3.5), we have

$$
\begin{aligned}
\theta \leq & x_{n+1} \oplus x_{n} \\
= & {\left[(1-\rho) x_{n}+\rho G_{1}\left(x_{n}, y_{n}\right)\right] \oplus\left[(1-\rho) x_{n-1}+\rho G_{1}\left(x_{n-1}, y_{n-1}\right)\right] } \\
\leq & (1-\rho)\left(x_{n-1} \oplus x_{n}\right)+\rho\left(G_{1}\left(x_{n}, y_{n}\right) \oplus G_{1}\left(x_{n-1}, y_{n-1}\right)\right) \\
\leq & (1-\rho)\left(x_{n-1} \oplus x_{n}\right)+\rho\left(G_{1}\left(x_{n}, y_{n}\right) \oplus G_{1}\left(x_{n-1}, y_{n-1}\right)\right) \\
\leq & {\left[(1-\rho)+\rho\left(\alpha_{1}\left(\mu_{11} \gamma_{f}+v_{12}+\zeta_{1}\right)+\alpha_{2}\right)\right]\left(x_{n-1} \oplus x_{n}\right) } \\
& +\rho \alpha_{1}\left(v_{11}+\mu_{12}\right)\left(y_{n-1} \oplus y_{n}\right)
\end{aligned}
$$

and

$$
\begin{aligned}
\theta & \leq y_{n+1} \oplus y_{n} \\
& =\left[(1-\sigma) y_{n}+\sigma G_{2}\left(x_{n}, y_{n}\right)\right] \oplus\left[(1-\sigma) y_{n-1}+\sigma G_{2}\left(x_{n-1}, y_{n-1}\right)\right] \\
& \leq(1-\sigma)\left(y_{n-1} \oplus y_{n}\right)+\sigma\left(G_{2}\left(x_{n}, y_{n}\right) \oplus G_{2}\left(x_{n-1}, y_{n-1}\right)\right) \\
& \leq(1-\sigma)\left(y_{n-1} \oplus y_{n}\right)
\end{aligned}
$$




$$
\begin{aligned}
& +\sigma\left(\beta_{1}\left(\mu_{21} \oplus \mu_{22}\right)\left(x_{n-1} \oplus x_{n}\right)+\left(\left(\beta_{1} v_{21} \oplus v_{22} \gamma_{g}\right)+\zeta_{2}+\beta_{2}\right)\left(y_{n-1} \oplus y_{n}\right)\right) \\
\leq & {\left[(1-\sigma)+\sigma\left(\left(\beta_{1} v_{21} \oplus v_{22} \gamma_{g}\right)+\zeta_{2}+\beta_{2}\right)\right]\left(y_{n-1} \oplus y_{n}\right) } \\
& +\sigma \beta_{1}\left(\mu_{21} \oplus \mu_{22}\right)\left(x_{n-1} \oplus x_{n}\right) \\
\leq & \sigma \beta_{1}\left(\mu_{21} \oplus \mu_{22}\right)\left(x_{n-1} \oplus x_{n}\right) \\
& +\left[(1-\sigma)+\sigma\left(\left(\beta_{1} v_{21} \oplus v_{22} \gamma_{g}\right)+\zeta_{2}+\beta_{2}\right)\right]\left(y_{n-1} \oplus y_{n}\right),
\end{aligned}
$$

combining (3.12) and (3.13), and by Definition 2.8, we have

$$
\left(x_{n+1}, y_{n+1}\right) \oplus\left(x_{n}, y_{n}\right) \leq \boldsymbol{\Sigma}\left(x_{n}, y_{n}\right) \oplus\left(x_{n-1}, y_{n-1}\right),
$$

where

$$
\begin{aligned}
\boldsymbol{\Sigma}= & \left(\begin{array}{cc}
1-\rho & 0 \\
0 & 1-\sigma
\end{array}\right) \\
& +\left(\begin{array}{cc}
\rho\left(\alpha_{1}\left(\mu_{11} \gamma_{f}+\nu_{12}+\zeta_{1}\right)+\alpha_{2}\right) & \rho \alpha_{1}\left(v_{11}+\mu_{12}\right) \\
\sigma \beta_{1}\left(\mu_{21} \oplus \mu_{22}\right) & \sigma\left(\left(\beta_{1} \nu_{21} \oplus \nu_{22} \gamma_{g}\right)+\zeta_{2}+\beta_{2}\right)
\end{array}\right) .
\end{aligned}
$$

Since (3.2) holds, that is,

$$
\begin{array}{r}
N \max \left\{\alpha_{1}\left(\mu_{11} \gamma_{f}+v_{12}+\zeta_{1}\right)+\alpha_{2}, \alpha_{1}\left(v_{11}+\mu_{12}\right),\right. \\
\left.\beta_{1}\left(\mu_{21} \oplus \mu_{22}\right),\left(\beta_{1} \nu_{21} \oplus v_{22} \gamma_{g}\right)+\zeta_{2}+\beta_{2}\right\}<1,
\end{array}
$$

the inequality $N\|\boldsymbol{\Sigma}\|<1$ is true. It follows that $\left(x_{n}, y_{n}\right)^{T} \rightarrow\left(x^{*}, y^{*}\right)^{T}$ strongly from Lemma 2.11 .

Since $g, f, B_{1}, B_{2}$ and $F_{i j}(i, j=1,2)$ are ordered compressions, and they are comparisons of each other, so that

$$
\begin{aligned}
& x^{*}=(1-\rho) x^{*}+\rho\left(\left(F_{11}\left(f\left(x^{*}\right), y^{*}\right)+F_{12}\left(y^{*}, x^{*}\right)-u\right) \wedge B_{1}\left(x^{*}\right)+I\left(x^{*}\right)\right), \\
& y^{\prime \prime}=(1-\sigma) y^{* \prime}+\sigma\left(\left(\left(F_{21}\left(x^{*}, y^{*}\right) \oplus F_{22}\left(x^{*}, g\left(y^{*}\right)\right)-v\right) \wedge B_{2}\left(y^{*}\right)+I\left(y^{*}\right)\right)\right)
\end{aligned}
$$

holds. Therefore, $\left(x^{*}, y^{*}\right)$ is a fixed point of the vector-valued mapping

$$
\left(\left(F_{11}(f(x), y)+F_{12}(y, x)-u\right) \wedge B_{1}(x)+I(x),\left(F_{21}(x, y) \oplus F_{22}(x, g(y))-v\right) \wedge B_{2}(y)+I(y)\right) .
$$

By using Lemma 3.1, $\left(x^{*}, y^{*}\right)$ is a solution of system (1.1). This completes the proof.

Remark 3.4 For a suitable choice of the mappings $g, f, B_{1}, B_{2}$ and $F_{i j}(i, j=1,2)$, we can obtain several known results [1] and [2] as special cases of Theorem 3.2, 3.3. 


\section{Author details}

'Applied Mathematics Institute, College of Mathematics and Physics, Chongqing University of Posts and Telecommunications, Chongqing, 400065, China. ${ }^{2}$ Institute of Nonlinear Analysis Research, Changjiang Normal University, Fuling, Chongqing, 400803, China.

\section{Acknowledgements}

This work was supported by the National Natural Science Foundation of China (Grant no. 11201512) and the Natural Science Foundation Project of CQ CSTC (cstc2012jjA00001).

\section{Received: 25 February 2013 Accepted: 27 August 2013 Published: 08 Nov 2013}

\section{References}

1. Li, HG: Approximation solution for general nonlinear ordered variational inequalities and ordered equations in ordered Banach space. Nonlinear Anal. Forum 13(2), 205-214 (2008)

2. Li, HG: Approximation solution for a new class of general nonlinear ordered variational inequalities and ordered equations in ordered Banach space. Nonlinear Anal. Forum 14, 1-9 (2009)

3. Verma, RU: Projection methods, algorithms and a new system of nonlinear variational inequalities. Comput. Math. Appl. 41, 1025-1031 (2001)

4. Amann, H: On the number of solutions of nonlinear equations in ordered Banach space. J. Funct. Anal. 11, 346-384 (1972)

5. Du, YH: Fixed points of increasing operators in ordered Banach spaces and applications. Appl. Anal. 38, 1-20 (1990)

6. Ge, DJ, Lakshmikantham, V: Couple fixed points of nonlinear operators with applications. Nonlinear Anal. TMA 11, 623-632 (1987)

7. Ge, DJ: Fixed points of mixed monotone operators with applications. Appl. Anal. 31, 215-224 (1988)

8. Li, HG: Nonlinear inclusion problems for ordered RME set-valued mappings in ordered Hilbert spaces. Nonlinear Funct. Anal. Appl. 16(1), 1-8 (2011)

9. Li, HG: Nonlinear inclusion problem involving $(\alpha, \lambda)$-NODM set-valued mappings in ordered Hilbert space. Appl. Math. Lett. 25, 1384-1388 (2012)

10. Ansari, QH, Yao, JC: A fixed point theorem and its applications to a system of variational inequalities. Bull. Aust. Math. Soc. 59, 433-442 (1999)

11. Ansari, QH, Schaible, S, Yao, JC: Systems of vector equilibrium problems and its applications. J. Optim. Theory Appl. $107,547-557(2000)$

12. Cho, YJ, Fang, YP, Huang, NJ: Algorithms for systems of nonlinear variational inequalities. J. Korean Math. Soc. 41 489-499 (2004)

13. Fang, YP, Huang, NJ: H-Monotone operators and system of variational inclusions. Commun. Appl. Nonlinear Anal. 11(1), 93-101 (2004)

14. Yan, WY, Fang, YP, Huang, NJ: A new system of set-valued variational inclusions with $H$-monotone operators. Math. Inequal. Appl. 8(3), 537-546 (2005)

15. Fang, YP, Huang, $N J$, Thompson, HB: A new system of variational inclusions with $(H, \eta)$-monotone operators in Hilbert spaces. Comput. Math. Appl. 49, 365-374 (2005)

16. Lan, HY, Kang, Jl, Cho, YJ: Nonlinear $(A, \eta)$-monotone operator inclusion systems involving non-monotone set-valued mappings. Taiwan. J. Math. 11, 683-701 (2007)

17. Peng, JW, Zhu, DL: Three-step iterative algorithm for a system of set-valued variational inclusions with $(H, \eta)$-monotone operators. Nonlinear Anal. 68, 139-153 (2008)

18. $\mathrm{Li}, \mathrm{HG}, \mathrm{Xu}, \mathrm{AJ}$, Jin, MM: A hybrid proximal point three-step algorithm for nonlinear set-valued quasi-variational inclusions system involving $(A, \eta)$-accretive mappings. Fixed Point Theory Appl. 2010, Article ID 635382 (2010). doi:10.1155/2010/635382

19. Lan, HY, Cho, YJ, Verma, RU: On nonlinear relaxed cocoercive inclusions involving $(A, \eta)$-accretive mappings in Banach spaces. Comput. Math. Appl. 51, 1529-1538 (2006)

20. Li, HG: Approximation solutions for generalized multi-valued variational-like inclusions with $(G, \eta)$-monotone mappings. J. Jishou Univ., Nat. Sci. Ed. 30(4), 7-12 (2009)

21. Schaefer, HH: Banach Lattices and Positive Operators. Springer, Berlin (1974)

22. Li, HG, Xu, AJ, Jin, MM: An Ishikawa-hybrid proximal point algorithm for nonlinear set-valued inclusions problem based on $(A, \eta)$-accretive framework. Fixed Point Theory Appl. 2010, Article ID 501293 (2010). doi:10.1155/2010/501293

23. Pan, $\mathrm{XB}, \mathrm{Li}, \mathrm{HG}, \mathrm{Xu}, \mathrm{AJ}$ : The over-relaxed A-proximal point algorithm for general nonlinear mixed set-valued inclusion framework. Fixed Point Theory Appl. 2011, Article ID 840978 (2011). doi:10.1155/2011/840978

24. Li, H-G, Xu, A-J: A new class of generalized nonlinear random set-valued quasi-variational inclusion system with random nonlinear $\left(\mathbf{A}_{i \omega}, \boldsymbol{\eta}_{i \omega}\right)$-accretive mappings in q-uniformly smooth Banach spaces. Nonlinear Anal. Forum 15 $1-20(2010)$

25. Shim, SH, Kang, SM, Huang, NJ, Yao, JC: Perturbed iterative algorithms with errors for completely generalized strongly nonlinear implicit variational-like inclusions. J. Inequal. Appl. 5(4), 381-395 (2000)

26. Verma, RU: A-Monotonicity and applications to nonlinear variational inclusions. J. Appl. Math. Stoch. Anal. 17(2) 193-195 (2004)

27. Horn, RA, Johnson, CR: Matrix Analysis. Cambridge University Press, Cambridge (1986)

28. Li, HG: Approximation solutions for generalized multi-valued variational-like inclusions with $(G, \eta)$-monotone mappings. J. Jishou Univ., Nat. Sci. Ed. 30(4), 7-12 (2009)

29. Alimohammady, M, Balooee, J, Cho, YJ, Roohi, M: Iterative algorithms for a new class of extended general nonconvex set-valued variational inequalities. Nonlinear Anal. 73, 3907-3923 (2010)

30. Alimohammady, M, Balooee, J, Cho, YJ, Roohi, M: New perturbed finite step iterative algorithms for a system of extended generalized nonlinear mixed-quasi variational inclusions. Comput. Math. Appl. 60, 2953-2970 (2010)

31. Yao, Y, Cho, YJ, Liou, Y: Iterative algorithms for variational inclusions, mixed equilibrium problems and fixed point problems approach to optimization problems. Cent. Eur. J. Math. 9, 640-656 (2011) 
32. Yao, Y, Cho, YJ, Liou, Y: Algorithms of common solutions for variational inclusions, mixed equilibrium problems and fixed point problems. Eur. J. Oper. Res. 212, 242-250 (2011)

10.1186/1029-242X-2013-514

Cite this article as: Li et al.: GNM ordered variational inequality system with ordered Lipschitz continuous mappings in an ordered Banach space. Journal of Inequalities and Applications 2013, 2013:514

Submit your manuscript to a SpringerOpen ${ }^{\circ}$ journal and benefit from:

- Convenient online submission

- Rigorous peer review

- Immediate publication on acceptance

Open access: articles freely available online

- High visibility within the field

- Retaining the copyright to your article

Submit your next manuscript at $>$ springeropen.com 